\title{
Effect of Dietary Lipids on Longevity and Memory in the SAMP8 Mice
}

\author{
Yukiko UEDA ${ }^{1}$, Ming-Fu WANG ${ }^{2}$, Amalia Veronica IREI ${ }^{3}$, Nobuko SARUKURA ${ }^{4}$, \\ Tohru SAKAI ${ }^{5}$ and Tzu-Fang HsU ${ }^{6}$ \\ ${ }^{1}$ Department of Food Science and Nutrition, Faculty of Human Life and Environmet, \\ Nara Women's University, Nara 630-8506, Japan \\ ${ }^{2}$ Department of Food and Nutrition, Providence University, Taichung, Taiwan \\ ${ }^{3}$ School of Nutrition, Faculty of Medicine, Universidad Abierta Interamericana, Buenos Aires, Argentina \\ ${ }^{4}$ National Institute of Health and Nutrition, Tokyo 162-8636, Japan \\ ${ }^{5}$ Graduate School of Nutrition and Biosciences, The University of Tokushima 770-8501, Japan \\ ${ }^{6}$ Hungkung University Applied Cosmetology and Graduate Institute of Cosmetic Science, \\ Chung-Chie Rd., Shalu, Taichung County, Taiwan
}

(Received June 15, 2010)

\begin{abstract}
Summary The senescence-accelerated mouse (SAMP8) is an animal model used in studies of aging. The purpose of this study was to investigate the effect of different types of dietary lipids on longevity and age-related deterioration in memory in SAMP8 mice. Eightmonth-old mice were fed diets with 5\% lard (Lard group), 5\% soybean oil (Soy group), $2 \%$ lecithin $+3 \%$ soybean oil (Lecithin + Soy group) or $2 \%$ fish oil $+3 \%$ soybean oil (Fish + Soy group), and learning and memory were examined by passive avoidance test at 8 and 10 mo of age. At the end of the experiment, the mice were killed, and the brain fatty acid composition was analyzed. The results indicated that the survival rates at 12 mo decreased in the order: Fish + Soy $>$ Soy $>$ Lecithin+Soy $>$ Lard and were 50, 40, 30 and 20\%, respectively. The rate of the lard group was lower than that of the Fish+Soy group, but this difference was not statistically significant. At $10 \mathrm{mo}$ of age, the passive avoidance times of the Fish + Soy, Lecithin + Soy, Soy and Lard groups were 166, 170, 149 and 127 s, respectively. The passive avoidance times of the Fish+Soy and Lecithin+Soy groups were longer than that of the Lard group $(p<0.01)$ at 10 mo. The brain DHA concentration was the highest in the Fish+Soy group SAMP8 mice, the linoleic acid levels was highest in the Soy group and the palmitic acid level was lowest in the Lard group $(p<0.05)$. In conclusion, compared with SAMP8 mice fed a high saturated fatty acid diet, SAMP8 mice given a high poly-unsaturated fatty acid diet had higher brain concentrations of poly-unsaturated acid, better memory and greater longevity.
\end{abstract}

Key Words dietary lipids, brain fatty acid, passive avoidance test, SAMP8 mice, aging

Senescence accelerated prone mice, such as SAMprone8 (SAMP8), exhibit several features that make them interesting models of human aging, including age-associated early onset of senile amyloidosis, degenerative arthropathy, cataracts, osteoporosis and osteoarthritis, reduced fecundity and early loss of fertility (1-3). SAMP8 mice have shortened life-spans and earlier manifestations of senescence, including learning and memory deterioration, as well as various pathological features of neurodegeneration observed early in life $(4,5)$. Thus, when used as a mouse analog of Alzheimer's disease (AD), SAMP8 models human brain aging.

Studies on types of dietary lipids in rats and mice (610 ), suggest that a dietary $n-6 / n-3$ ratio affects lifespan. However, the saturated : monounsaturated : polyunsaturated fatty acid ratio ( $\mathrm{S}: \mathrm{M}: \mathrm{P}$ ratio) of the test fats used in these experiments was not regulated. Thus,

E-mail: yueda@life.osaka-cu.ac.jp it is not clear whether the lipid-type influence on lifespan was caused by the $n-6 / n-3$ ratio.

Epidemiological (11-13) and animal studies (14-17) report an association between learning ability and dietary intake of $n$-3 PUFA. A balance between dietary $n-6$ PUFA and $n-3$ PUFA affects the ratio between $n-6$ PUFA and $n$-3 PUFA in the brain (PL) of 7 -mo-old SAMP8 mice. These studies suggest a mechanism by which dietary PUFA influences learning and memory (18).

Previous studies have shown that a diet heavy in butter, saturated fatty acids, or fish oil with $n$-3 PUFAs, advances the severity of age-related senile amyloidosis, whereas soybeans enriched with $n-6$ PUFAs alleviate the severity of the age-related disease (19). These findings are contrary to the popular hypothesis that n-3 PUFAs are beneficial to health.

In the present study, we investigated the effect of different types of lipids on longevity, brain lipid fatty acid and the age-related deterioration of learning and memory in SAMP8 mice. 
Table 1. Composition of the experimental diets $(\mathrm{g} / \mathrm{kg}$ diet). ${ }^{1}$

\begin{tabular}{|c|c|c|c|c|}
\hline Ingredients & Fish + Soy & Lecithin + Soy & Soy & Lard \\
\hline Casein $^{2}$ & 200 & 200 & 200 & 200 \\
\hline$\alpha$-Starch & 454 & 454 & 454 & 454 \\
\hline Sucrose & 226 & 226 & 226 & 226 \\
\hline Mineral mix $^{3}$ & 40 & 40 & 40 & 40 \\
\hline Vitamin $\operatorname{mix}^{4}$ & 10 & 10 & 10 & 10 \\
\hline Cellulose & 20 & 20 & 20 & 20 \\
\hline Soybean oil & 30 & 30 & 50 & - \\
\hline Lard & - & - & - & 50 \\
\hline Fish oil ${ }^{5}$ & 20 & - & - & - \\
\hline Lecithin $^{5}$ & - & 20 & - & - \\
\hline
\end{tabular}

${ }^{1}$ Fish +Soy group: $2 \%$ fish oil $+3 \%$ soybean oil; Lecithin +Soy group: $2 \%$ lecithin $+3 \%$ soybean oil; Soy group: 5\% soybean oil; Lard group: 5\% lard.

${ }^{2} 82.3 \%$ crude protein, Oriental Yeast Co., Ltd. (Tokyo, Japan).

${ }^{3}$ The mineral composition was $(\mathrm{g} \%) 14.56 \mathrm{CaHPO}_{4}$. $2 \mathrm{H}_{2} \mathrm{O}, 25.72 \mathrm{KH}_{2} \mathrm{PO}_{4}, 9.35 \mathrm{NaH}_{2} \mathrm{PO}_{4}, 4.66 \mathrm{NaCl}, 35.09$ Ca-lactate, $3.18 \mathrm{Fe}$-citrate, $7.17 \mathrm{MgSO}_{4}, 0.11 \mathrm{ZnCO}_{3}$, $0.12 \mathrm{MnSO}_{4} \cdot 5 \mathrm{H}_{2} \mathrm{O}, 0.03 \mathrm{CuSO}_{4} \cdot 5 \mathrm{H}_{2} \mathrm{O}$ and $0.01 \mathrm{KI}$; Kewpie Corporation, Japan.

${ }^{4}$ The vitamin composition $(\mathrm{mg} \%)$ was 100 retinyl acetate, 0.25 ergocalciferol, 500 tocopheryl acetate, 520 menadione, 120 thiamine- $\mathrm{HCl}, 400$ riboflavin, 80 vita$\min \mathrm{B}_{6}-\mathrm{HCl}, 0.05$ vitamin $\mathrm{B}_{12}$, 3,000 ascorbic acid, 2.0 D-biotin, 20 folic acid, 500 Ca-pantothenate, 500 p-aminobenzoic acid, 600 niacin, 600 inositol, 2,000 choline choride, cellulose up to 73,057.7; Kewpie Corporation, Japan.

${ }^{5}$ Kewpie Corporation, Japan.

\section{MATERIALS AND METHODS}

Animals and diets. Male SAMP8 mice of $8 \mathrm{mo}$ $(n=71)$ were used as a murine model of aging in this experiment. The mice were housed under controlled environmental conditions $\left(23 \pm 2^{\circ} \mathrm{C}, 65 \pm 5 \%\right.$ relative humidity, $12 \mathrm{~h}$ dark-light cycles). The mice were divided into four groups according to the administered dietary lipid as follows: $2 \%$ lecithin $+3 \%$ soybean oil (Lecithin + Soy group), $2 \%$ fish oil $+3 \%$ soybean oil (Fish+Soy group), 5\% soybean oil (Soy group), or 5\% lard (Lard group). The composition of the experimental diet is shown in Tables 1 and 2. Eight-month-old mice were allowed free access to food and water during the experimental period. The study protocol was approved by the Animal Research Ethics Committee at Providence University, Taiwan.

Memory examination. The mice were subjected to step-through passive avoidance tests as described in a prior study $(20)$. The cage $[35 \times 17 \times 20 \mathrm{~cm}$ (width $\times$ length $\times$ height), model E10-15; Coulbourn Instruments] consisted of two equal compartments connected by a small guillotine door $(7.5 \times 6.5 \mathrm{~cm}$ guillotine door, model E10-15GD; Coulbourn Instruments). One of the compartments was lighted, and the other was darkened by covering the top with a black semitransparent plastic. The floor was a platform of steel rods. Each mouse
Table 2. Fatty acid (FA) composition of the experimental diets.

\begin{tabular}{lccrr}
\hline \multicolumn{1}{c}{ Fatty acid (\%) } & Fish+Soy & Lecithin+Soy & Soy & Lard \\
\hline SFA & & & & \\
16:0 & 11.9 & 14.5 & 9.9 & 22.2 \\
18:0 & 3.8 & 6.1 & 3.9 & 6.6 \\
MUFA & & & & \\
16:1 & 1.9 & 0.4 & 0.1 & 3.4 \\
18:1 & 22.6 & 22.9 & 20.7 & 51.4 \\
20:1 & & & & 1.2 \\
$n-6$ PUFA & & & & \\
18:2n-6 (LA) & 38.2 & 48.2 & 56.9 & 13.9 \\
20:2n-6 & & & & 0.6 \\
20:4n-6 (AA) & 1.0 & 1.2 & & \\
$n-3$ PUFA & & & & \\
18:3n-3 (ALA) & 5.5 & 6.7 & 8.5 & 0.7 \\
20:5n-3 (EPA) & 3.1 & & & \\
22:5n-3 (DPA) & 0.9 & & & \\
22:6n-3 (DHA) & 11.0 & & & \\
\end{tabular}

LA: linoleic acid; AA: arachidonic acid; ALA: $\alpha$-linolenic acid; EPA: eicosapentaenoic acid; DPA: docosapetaenoic acid; DHA: docosahexaenoic acid.

exposed to this test was placed in the lighted compartment and, after a brief orientation period (10 s), the partition was raised, allowing the mouse to freely explore the apparatus. Once the mouse was inside the dark compartment, the partition was closed, and mice received a punishing electrical shock (three 50-V shocks, $0.5 \mathrm{~s}$ each). Mice were placed in the lighted compartment on days 1 and 7 after the punishment, and the latency (seconds to enter the dark compartment and avoid punishment) was recorded. The optimal latency was defined as $180 \mathrm{~s}$.

Brain fatty acid analysis. Brain tissue was removed for fatty acid analysis at 12 mo. Total lipids were extracted using chloroform/methanol (2:1 vol/vol) following the Folch method (21) to increase the final volume 20 times, and butylated hydroxianisole (BHA, $0.002 \%$ ) was added as an antioxidant. The sample was homogenized, mixed with saline $(0.58 \% \mathrm{NaCl})$ at room temperature and centrifuged (3,000 rpm, $10 \mathrm{~min})$. The supernatant was collected, evaporated under a nitrogen stream and subjected to methylation. The methylated lipid sample was then saponified by $6 \% \mathrm{~K}_{2} \mathrm{CO}_{3}$, mixed with hexane and extracted. The extracted sample was dried under a nitrogen stream, and then subjected to a Hitachi gas chromatographer Model 5000 equipped with a flame ionization detector and an automatic sampler (Hitachi Ltd., Tokyo, Japan), a $30 \mathrm{~m} \times 0.25 \mathrm{~mm}$ fused silica capillary column (SP 2330, J \& W Scientific, CA, USA) and a Hitachi D-2500 chromato-integrator (Hitachi Ltd.).

Fatty Acid Analysis of the experimental diets. Each sample was analyzed for fatty acid composition by gas liquid chromatography as described above.

Statistical analyses. The differences between groups were evaluated using ANOVA, followed by post-hoc 
Table 3. Body weights and food intakes in 8-mo-old SAMP8 mice fed different diets for 16 wk.

\begin{tabular}{|c|c|c|c|c|c|c|c|c|c|}
\hline \multirow{3}{*}{ Group } & \multirow{3}{*}{$n$} & \multicolumn{6}{|c|}{ Body weight (g) } & \multirow{2}{*}{\multicolumn{2}{|c|}{$\begin{array}{l}\text { Food intake } \\
\qquad(\mathrm{g} / \mathrm{d})\end{array}$}} \\
\hline & & \multicolumn{2}{|c|}{$8 \mathrm{mo}$} & \multicolumn{2}{|c|}{$10 \mathrm{mo}$} & \multicolumn{2}{|c|}{$12 \mathrm{mo}$} & & \\
\hline & & Mean & $\mathrm{SD}$ & Mean & $\mathrm{SD}$ & Mean & SD & Mean & SD \\
\hline Fish +Soy & 20 & $28.3^{\mathrm{a}}$ & 0.6 & $29.9^{a}$ & 1.1 & $29.5^{\mathrm{a}}$ & 1.1 & 5.24 & 0.19 \\
\hline Lecithin + Soy & 18 & $27.9^{\mathrm{a}}$ & 0.8 & $26.4^{\mathrm{b}}$ & 2.9 & $27.6^{b}$ & 0.8 & 5.56 & 1.13 \\
\hline Soy & 19 & $29.6^{b}$ & 0.6 & $28.8^{\mathrm{a}}$ & 0.8 & $27.9^{b}$ & 0.5 & 5.24 & 0.30 \\
\hline Lard & 14 & $25.1^{\mathrm{c}}$ & 1.2 & $30.9^{\mathrm{a}}$ & 1.1 & $31.6^{\mathrm{c}}$ & 0.6 & 5.97 & 1.16 \\
\hline
\end{tabular}

Values are mean \pm SD.

${ }^{\mathrm{a}-\mathrm{c}}$ Means with different superscripts in a column are significantly based on the ANOVA $(p<0.05)$ test.

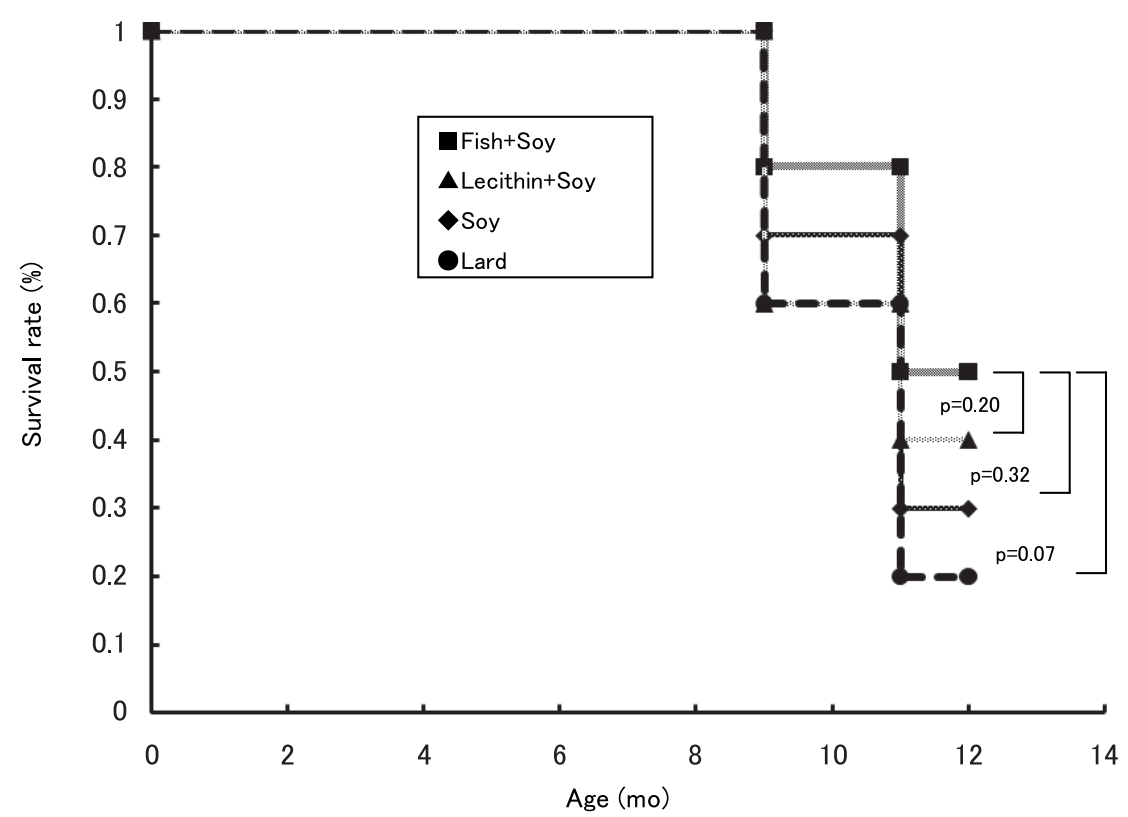

Fig 1. Survival rates in 12-mo-old SAMP8 fed diets with different lipids. Survival rate was estimated using Kaplan-Meier log rank analysis.

Scheffe tests. The survival rates were estimated using Kaplan-Meier long rank analysis. The results are expressed as mean \pm SD. A $p$-value $<0.05$ was considered statistically significant. SPSS 15.0 software (SPSS Inc., Chicago, IL, USA) was used to perform all statistical analyses.

\section{RESULTS}

Food intake and body weight

Changes in food intake and body weight are shown in Table 3. At baseline, significant differences were observed in food intake only in mice fed lard as the dietary lipid. Likewise, at baseline, significant differences were not observed among the four groups. SAMP8 mice in the Lard group showed the lowest weight at baseline. Effect of dietary lipids on survival rate

Figure 1 shows the survival rates of the SAMP8 mouse types for each experimental diet. There was no significant effect of diet on the survival rates (based on the log rank test, Fig. 1), but the highest survival rate in the SAMP8 mice was observed in the Fish+Soy group.
The survival rate at 12 mo was highest in the Fish+Soy group and was the lowest in the Lard group (Fish+Soy vs. Lard $p=0.07$ ).

Effect of dietary lipids in cognitive ability

The passive avoidance test was carried out on days 1 and 7 after a punishing electrical shock, and the latency was measured at 8 and 10 mo age (Fig. 2). For latency on day- 1 , the latency significantly declined in the Lard group at 10 mo but not at $8 \mathrm{mo}$. The passive avoidance time in the Fish+Soy and Lecithin+Soy groups were significantly $(p<0.01)$ longer than the time in the Lard group. However, the Fish+Soy and Lecithin + Soy groups did not show improvements in the passive avoidance time on day 7 .

Effect of dietary fatty acids on brain fatty acid

To evaluate the impact of dietary lipids on brain fatty acid composition, brain tissue was obtained at the end of the study, and fatty acids were measured (Table 4). As expected, DHA deposition was increased in the Fish + Soy group $(p<0.05)$ compared with all other dietary lipids. However, there were no differences in the 
Table 4. Fatty acid composition in brain lipids of SAMP8 mice along the experimental period.

\begin{tabular}{|c|c|c|c|c|}
\hline Fatty acid composition & Fish (10) & Lecithin (5) & Soy $(7)$ & Lard (3) \\
\hline 16:0 & $21.74 \pm 1.20^{\mathrm{a}}$ & $21.44 \pm 0.18^{\mathrm{ab}}$ & $21.01 \pm 1.38^{\mathrm{b}}$ & $20.16 \pm 0.29^{c}$ \\
\hline $16: 1$ & $0.68 \pm 0.06$ & $0.63 \pm 0.32$ & $0.63 \pm 0.07$ & $0.58 \pm 0.20$ \\
\hline 18:0 & $21.02 \pm 1.67$ & $21.07 \pm 0.86$ & $20.63 \pm 1.47$ & $21.83 \pm 2.23$ \\
\hline $18: 1$ & $20.45 \pm 4.27$ & $21.00 \pm 1.62$ & $21.68 \pm 3.44$ & $22.13 \pm 0.66$ \\
\hline $18: 2 n-6$ & $0.58 \pm 0.56^{b}$ & $0.54 \pm 0.13^{b}$ & $0.68 \pm 0.52^{\mathrm{a}}$ & $0.34 \pm 0.31^{\mathrm{c}}$ \\
\hline $18: 3 n-3$ & $3.26 \pm 0.54$ & $3.19 \pm 0.33$ & $3.50 \pm 0.41$ & $2.57 \pm 0.90$ \\
\hline $20: 2 n-6$ & $0.52 \pm 0.08$ & $0.51 \pm 0.11$ & $0.42 \pm 0.10$ & $0.42 \pm 0.18$ \\
\hline $20: 4 n-6$ & $9.08 \pm 0.55^{\mathrm{b}}$ & $10.75 \pm 0.95^{\mathrm{a}}$ & $10.82 \pm 0.75^{\mathrm{a}}$ & $10.59 \pm 0.64^{\mathrm{a}}$ \\
\hline $20: 5 n-3$ & $0.16 \pm 0.07$ & $0.44 \pm 0.03$ & $0.20 \pm 0.13$ & ND \\
\hline $22: 6 n-3$ & $21.49 \pm 1.28^{\mathrm{a}}$ & $19.62 \pm 0.19^{b}$ & $19.32 \pm 1.18^{b}$ & $18.49 \pm 0.66^{b}$ \\
\hline
\end{tabular}

${ }^{\mathrm{a}-\mathrm{c}}$ Means \pm SD (number of mice) for each experiment in row with different superscripts are significantly different by ANOVA $(p<0.05)$.

ND: not detected.
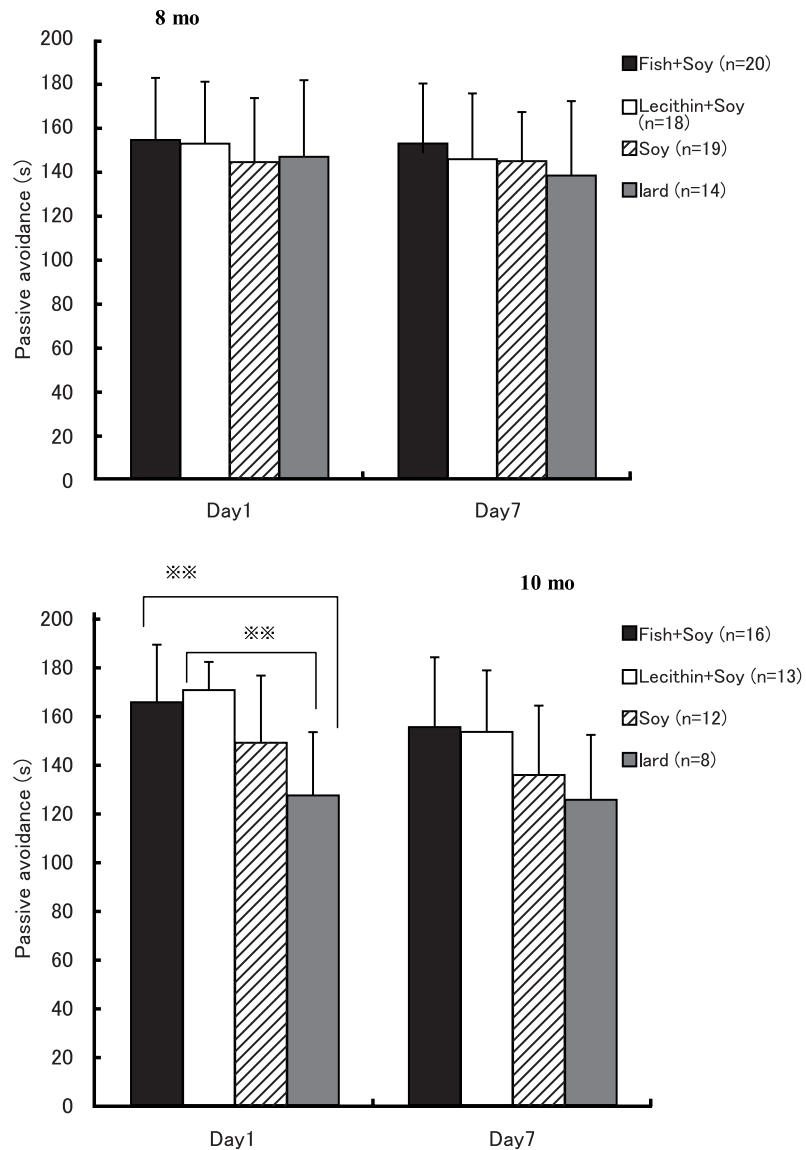

Fig 2. Memory measured by passive avoidance test in 8- and 10-mo-old SAMP8 fed diets with different lipids. Each value is the mean \pm SD. Avoidance tests were performed on the first day as well as on the seventh day of the study. There are significant differences between the Fish + Soy, Lecithin +Soy and Lard groups based on the one-way ANOVA (ANOVA followed by the Scheffe test; $* * p<0.01)$.

DHA levels among the Lecithin+Soy, Soy and Lard groups. DHA levels were higher and ARA levels were lower in the Fish +Soy group. The linoleic acid level was highest in the Soy group and the palmitic acid level was lowest in the Lard group $(p<0.05)$.

\section{DISCUSSION}

In the present study, we investigated the effect of different types of lipids (Fish+Soy, Lecithin + Soy, Soy, and Lard) on longevity, brain lipid fatty acid and the agerelated deterioration of learning and memory in SAMP8 mice. We demonstrated that marine DHA is incorporated into brain PL, and a diet of $n-3$ PUFA is associated with protection against a decline in learning and memory. Foods high in PUFA are associated with increased longevity. However, statistical differences were retained, and it is not clear whether the type of fatty acid in the feed was associated with the life-span of mice.

Takeuti et al. showed that variation in the $n-6 / n-3$ dietary ratio between 1 and 16 did not affect Wistar rat life-span (20). Indeed, no consistent findings have been reported with regard to the influence of dietary fat on life-span. Du et al. observed that the life-span of mice fed a DHA-rich diet was longer than that of mice fed a lard diet high in saturated fatty acids or a safflower oil diet rich in linoleic acid (6). However, Jolly et al. reported that mouse life-span in an autoimmune model was prolonged when fed fish oil rich in n-3 fatty acid, compared to a group fed a corn diet (7). Furthermore, non-obese NIDDM rats, hyperlipidemia model mice and senescence-accelerated mice have shorter life-spans than animals fed diets rich in $n-3$ fatty acids and those fed a diet rich in linoleic acid safflower oil $(8,9)$. Ratnayake et al. found a positive correlation between lifespan and a diet high in saturated fatty acids in spontaneously stroke-prone hypertensive rats, but not between life-span and diets with mono-, $n-6$, or $n-3$ unsaturated fatty acids (10). Based on these reports, the relationship between different fatty acid types and longevity is inconsistent. One reason for this inconsistency is that the effects of dietary fatty acids on life-span may vary among animal species or disease models, or by the type of lipids.

The passive avoidance times in the Fish+Soy and Lecithin + Soy groups were significantly longer than the Lard group $(p<0.01)$. These data suggest that 
Fish + Soy and Lecithin + Soy consumption improves mouse cognitive capacities, which may be relevant for age-related dementia. The effect of different types of lipids at the beginning of 8 mo was not significant. However, we found a significant difference between 10-moold mice, and others have shown that the Fish+Soy and Lecithin + Soy groups have improved protection against declines in learning and memory compared to the Soy group. In particular, the Fish + Soy group had the highest value, and the ratio of $n-6 / n-3$ may account for these differences. Besides, Yagi et al. reported similar retention impairment for passive avoidance training, but the deficit was not detectable until their P8 mice were at least 8 mo of age (21). Memory deterioration among mice at 8 mo was not remarkable, and thus, dietary fat was not expected to influence memory.

Moreover, the fatty acid composition in brain tissue mirrors the lipids in the assigned diets. The highest DHA deposition in brain tissue (22:6n-3) was observed in the Fish + Soy group. DHA is formed from $\alpha$-linolenic acid (18:3n-3, ALA), an essential fatty acid that is obtained either from the diet, or directly by consuming foods rich in DHA, such as fish or fish oil. The Lard group had no DHA. Minami et al. reported that fish oil, including fish oil high in DHA, maintains learning ability (22). Petursdottir et al. showed that dietary DHA maintains the phospholipids that protect the hippocampus and improves learning and memory. It was also demonstrated that a low $n-6 / n-3$ ratio is associated with improvement or protection of learning and memory (23). Additionally, foods rich in DHA may ameliorate learning and memory decline by protecting against the damage from amyloid accumulation or protein oxidation. These reports suggest that fish oil improves learning and memory. However, no significant differences in brain DHA were found between the Lecithin+Soy and Soy groups in the current study. The Soy group showed the worst memory (though the difference was non-significant) among all groups at all ages (despite the fact that the Lecithin+Soy and Soy groups had similar $n-$ $6: n-3$ ratios). Then, there were no differences in DHA deposition, $n-3 / n-6$ or 22:6n-3/20:4n-6 ratios in brain tissue between the Lecithin +Soy and Soy groups. The Lecithin+Soy group diet contained 2\% lecithin, a major source of choline, which improves acetylcholine concentration within the brain. Though all diets included choline mixed with other micronutrients, the Lecithin + Soy group also contained phosphatidylcholine (lecithin) as an extra ingredient to enhance the production of acetylcholine and thereby improve memory.

This study suggests that SAMP8 mice fed a diet high in PUFA had higher brain concentrations of poly-unsaturated acids and better memories, and tended to live longer than SAMP8 mice fed a diet high in saturated fatty acids. Fish and Soy or Lecithin and Soy consumption may improve the learning and cognitive capacities of mice, and these animal data are relevant for the field of age-related dementia. However, the Fish+Soy and Lecithin + Soy groups did not show improvements in the passive avoidance time on day 7 after testing at 10 mo in this study. Future studies are necessary to thoroughly understand the mechanisms involved in the affect of different types of lipids on cognitive impairment.

\section{Acknowledgments}

We are grateful to Dr. Cheng-Chung Lin of the Institute of Veterinary Pathology, National Chung Hsing University, for excellent technical assistance on the morphological examination.

\section{REFERENCES}

1) Takeda T. 1999. Senescence-accelerated mouse (SAM): a biogerontological resource in aging research. Neurobiol Aging 20: 105-110.

2) Takeda T, Hosokawa M, Higuchi K. 1997. Senescenceaccelerated mouse (SAM): a novel murine model of senescence. Exp Gerontol 32(1-2): 105-109.

3) Higuchi K, Matsumura A, Honma A, Toda K, Takeshita S, Matsushita M, Yonezu T, Hosokawa M, Takeda T. 1984. Age-related changes of serum apoprotein SASSAM, apoprotein A-I and low-density lipoprotein levels in senescence accelerated mouse (SAM). Mech Ageing Dev 26: 311-326.

4) Miyamoto M, Kiyota Y, Yamazaki N, Nagaoka A, Matsuo T, Nagawa Y, Takeda T. 1986. Age-related changes in learning and memory in the senescence-accelerated mouse. Physiol Behav 38: 399-406.

5) Nomura Y, Okuma Y. 1999. Age-related defects in lifespan and learning ability in SAMP8 mice. Neurobiol Aging 20: 111-115.

6) Du C, Sato A, Watanabe S, Ikemoto A, Fujii Y, Okuyama H. 2001. Effect of dietary oils enriched with $n$-3 fatty acids on survival of mice. J Nutr Biochem 12: 474-480.

7) Jolly CA, Muthukumar A, Avula CP, Troyer D, Fernandes G. 2001. Life span is prolonged in foodrestricted autoimmune-prone $(\mathrm{NZB} \times \mathrm{NZW}) \mathrm{F}(1)$ mice fed a diet enriched with (n-3) fatty acids. J Nutr 131: 2753-2760.

8) Berdanier CD, Johnson B, Hartle DK, Crowell W. 1992. Life span is shortened in BHE/cdb rats fed a diet containing 9\% menhaden oil and $1 \%$ corn oil. J Nutr 122: 1309-1317.

9) Umezawa M, Takeda T, Kogishi K, Higuchi K, Matushita T, Wang J, Chiba T, Hosokawa M. 2000. Serum lipid concentrations and mean life span are modulated by dietary polyunsaturated fatty acids in the senescenceaccelerated mouse. J Nutr 130: 221-227.

10) Ratnayake WM, Plouffe L, Hollywood R, L'Abbe MR, Hidiroglou N, Sarwar G, Mueller R. 2000. Influence of sources of dietary oils on the life span of stroke-prone spontaneously hypertensive rats. Lipids 35: 409-420.

11) Kalmijn S. 2000. Fatty acid intake and the risk of dementia and cognitive decline: a review of clinical and epidemiological studies. J Nutr Health Aging 4: 202207.

12) Kalmijn S, van Boxtel MP, Ocke M, Verschuren WM, Kromhout D, Launer LJ. 2004. Dietary intake of fatty acids and fish in relation to cognitive performance at middle age. Neurology 62: 275-280.

13) Van Gelder BM, Tijhuis M, Kalmijn S, Kroumhout D. 2007. Fish consumption, $n-3$ fatty acids, and subsequent 5-y cognitive decline in elderly men: the Zutphen 
Elderly Study. Am J Clin Nutr 85: 1142-1147.

14) Yamamoto N, Saitoh M, Moriuchi A, Nomura M, Okuyama H. 1987. Effect of dietary alpha-linolenate/ linoleate balance on brain lipid compositions and learning ability of rats. J Lipid Res 28: 144-151.

15) Suzuki H, Park SJ, Tamura M, Ando S. 1998. Effect of the long-term feeding of dietary lipids on the learning ability, fatty acid composition of brain stem phospholipids and synaptic membrane fluidity in adult mice: a comparison of sardine oil diet with palm oil diet. Mech Ageing Dev 101: 119-128.

16) Gamoh S, Hashimoto M, Sugioka K. 1999. Chronic administration of docosahexaenoic acid improves regerence memory-related learning ability in young rats. Neuroscience 93: 237-241.

17) Carrie I, Guesnet P, Bourre J-M, Frances H. 2000. Diets containing long-chain $n$-3 polyunsaturated fatty acids affect behaviour differently during development than ageing in mice. Br J Nutr 83: 439-447.

18) Umezawa M, Ohta A, Tojo H, Yagi H, Hosokawa M, Takeda T. 1995. Dietary $\alpha$-linolenate/linoleate balance influences learning and memory in the senescenceaccelerated mouse (SAM). Brain Res 669: 225-233.

19) Umezawa M, Tatematsu K, Korenaga T, Fu X, Matushita T, Okuyama H, Hosokawa M, Takeda T, Higuchi K.
2003. Dietary fat modulation of apoA-II metabolism and prevention of senile amyloidosis in the senescenceaccelerated mouse. J Lipid Res 44: 762-769.

20) Takeuti H, Sekine S, Noguchi O, Murano Y, Aoyama T, Matsuo T. 2009. Effect of life-long dierary $n-6 / n-3$ fatty acid ratio on life span, serum lipids and serum glucose in wistar rats. J Nutr Sci Vitaminol 55: 394-399.

21) Yagi H, Irino $M$, Matsushita $T$, Katoh $S$, Umezawa $M$, Tsuboyama T, Hosokawa M, Akiguchi I, Tokunaga R, Takeda T. 1989. Spontaneous spongy degeneration of the brain stem in SAM-P/8 mice, a newly developed memory-deficient strain. J Neuropathol Exp Neurol 48: 577-590.

22) Minami M, Kimura S, Endo T, Hamaue N, Hirafuji M, Togashi H, Matsumoto M, Yoshioka M, Saito H, Watanabe S, Kobayashi T, Okuyama H. 1997. Dietary docosahexaenoic acid increases cerebral acetylcholine levels and improves passive avoidance performance in stroke-prone spontaneously hypertensive rats. Pharmacol Biochem Behav 58: 1123-1129.

23) Petursdottir AL, Farr SA, Morley JE, Banks WA, Skuladottir GV. 2008. Effect of dietary n-3 polyunsaturated fatty acids on brain lipid fatty acid composition, learning ability, and memory of senescence-accelerated mouse. J Gerontol A Bio Sci Med Sci 63: 1153-1160. 